\title{
Equilibrium phase behavior of the membrane forming water-DMSO-EVAL copolymer system
}

\author{
Tai-Horng Young ${ }^{a}$, Juin-Yih Lai ${ }^{b}$, Wei-Ming You ${ }^{c}$, Liao-Ping Cheng ${ }^{c, *}$ \\ ${ }^{a}$ Center for Biomedical Engineering, College of Medicine, National Taiwan University, Taipei, Taiwan, ROC \\ ${ }^{\mathrm{b}}$ Department of Chemical Engineering, Chung Yuan University, Chung Li, Taiwan, ROC \\ ${ }^{\mathrm{c}}$ Department of Chemical Engineering, Tamkang University, Tamsui, Taipei Hsien, Taiwan, ROC
}

Received 10 June 1996; revised 18 October 1996; accepted 18 October 1996

\begin{abstract}
The equilibrium phase behavior of ethylene vinyl alcohol (EVAL) copolymer in mixtures of DMSO (dimethylsulfoxide, solvent) and water (nonsolvent) was studied for different temperatures. Both crystallization-induced gelation and liquid-liquid demixing were observed. From the determined phase diagram of this system at $25^{\circ} \mathrm{C}$, three regions may be identified, i.e., a homogeneous region, a gel region, and binodal region in which both types of phase transition take place. At higher temperatures, crystallization isotherm was found to intersect the binodal phase boundary, which is analogous to the phase behavior reported by Stokes and Berghmans for several binary systems.
\end{abstract}

Keywords: Poly(ethylene-co-vinyl alcohol); EVAL copolymer; Phase diagram; Crystallization; Liquid-liquid demixing; Interaction parameter

\section{Introduction}

The mechanism of membrane formation has been extensively studied for the past few decades. Various models were proposed, in which the aspects of equilibrium phase behavior and its relation to membrane morphology and permeative/selective characteristics were most frequently addressed [1-5]. Porous membranes are usually prepared by the immersion-precipitation process and the major forms of phase transformation of this process include liquid-liquid demixing and crystallization [5-8]. Polymer precipitated by liquid-liquid demixing is known to produce

${ }^{*}$ Corresponding author. membranes with open or closed cellular structures [1-8]. The role of crystallization in the precipitation process was, however, often overlooked and the equilibrium phase behavior of crystallization was rarely discussed. Until recently, some independent research of Cheng and Bulet reported observation of spherulitic crystallization of aliphatic polyamides from waterformic acid solutions [5,8]. The equilibrium phase behavior of water-formic acid-Nylons systems were studied by Cheng et al. [9]. Both equilibrium crystallization isotherm and binodal phase envelop were obtained. Following Cheng's approach, this work provides complete phase diagram of the ternary system, water-DMSO-poly(ethylene-co-vinyl alcohol) (EVAL). 
In 1979, Yamashita et al. utilized EVAL copolymer to produce membranes having improved permeability characteristics for the dialysis of blood [10]. Later, Sakurada et al. reported that using EVAL membrane in hemodialysis, efficient nonanticoagulant hemodialysis for renal failure patients was achieved [11]. Recently, EVAL copolymer membrane has attracted plenty of research interest in multiple fields of biomedical science [12-19]. In this current research, the isothermal phase behavior of EVAL in mixtures of dimethylsulfoxide (DMSO) and water (nonsolvent) was investigated for the temperature range between $25^{\circ} \mathrm{C}$ and $85^{\circ} \mathrm{C}$. Since EVAL copolymer is a crystalline polymer $[20,21]$, it is rather difficult to determine the liquid-liquid demixing phase envelop using cloud point data (the composition at which the turbidity changes) along. In a previous publication [12], cloud points measured by a titration method were given for water-DMSO-EVAL system at room temperature for a wide concentration range. These data differ somewhat from the present experimental results. In our opinion, this is caused by the fact that crystallization requires a considerably longer induction time than what was given for solutions to precipitate in the previous titration experiments. The data in this report are considered to be true equilibrium points and will be discussed in relation to the formation and properties of EVAL membranes. In addition, the variation of the onset of phase transformation with temperature, which leads to opalescence and separated layers in the solution, will be discussed.

\section{Theory}

\subsection{The liquid-liquid phase equilibrium}

At equilibrium between two phases ( $\alpha$ and $\beta$ ) in the ternary system at a specified temperature and pressure, the chemical potential of each component in these two phases are equal [22], i.e.,

$\mu_{i}=\mu_{i}^{\beta} \quad(i=1,2,3)$

where $\mu_{i}^{\alpha}$ and $\mu_{i}^{\beta}$ are the chemical potentials of component $i$ in phases $\alpha$ and $\beta$. In this report components 1,2 , and 3 denotes, respectively, nonsolvent, solvent and polymer. Expressing the concentration dependence of Flory-Huggins interaction parameters $\left(\chi_{12}, \chi_{13}\right.$, and $\left.\chi_{23}\right)$ [23] in Eq. (1) in different forms, Altena [24], McHugh [25] and Cheng [9] have formulated $\mu_{i}^{\alpha}$ and $\mu_{i}^{\beta}$ as a function of volume fraction and molar volume of each component. Since volume fractions of all components add up to 1 in both phases, for each phase

$\phi_{1}^{\alpha}+\phi_{2}^{\alpha}+\phi_{3}^{\alpha}=1$
$\phi_{1}^{\beta}+\phi_{2}^{\beta}+\phi_{3}^{\beta}=1$

Eqs. (1-3) describe the composition of the equilibrium phases. Given interaction parameters and molar volumes, these equations were used to compute the binodal of the water-DMSO-EVAL system in this report.

\subsection{The crystallization equilibrium}

As an equilibrium is established between the liquid phase and the polymer crystal in a ternary system, the chemical potentials per repeating unit of polymer are equal in both phases. It follows that [23]:

$\mathrm{d} \mu_{\gamma}=\mathrm{d} \mu_{\delta}$

where $\mu_{\gamma}$ and $\mu_{\delta}$ are the chemical potentials of polymer per repeating unit in phases $\gamma$ and $\delta$. Using a modified Flory-Huggins expression for the chemical potential and a temperature-dependent enthalpy of fusion, Cheng has derived an equation for the crystallization equilibrium [9]:

$$
\begin{aligned}
& C\left(1-\frac{T}{T_{\mathrm{m}}}\right)-A T \ln \frac{T}{T_{\mathrm{m}}}-\frac{B}{2}\left(T^{2}-T T_{\mathrm{m}}\right) \\
& +\frac{V_{\mathrm{u}}}{V_{3}} \ln \phi_{3}+\frac{V_{\mathrm{u}}}{V_{3}}\left(1-\phi_{3}\right)-\frac{V_{\mathrm{u}}}{V_{1}} \phi_{1}-\frac{V_{\mathrm{u}}}{V_{2}} \phi_{2} \\
& +\left(\frac{V_{\mathrm{u}}}{V_{1}} \phi_{1} \chi_{13}+\frac{V_{\mathrm{u}}}{V_{2}} \phi_{2} \chi_{23}\right)\left(\phi_{1}+\phi_{2}\right)-\frac{V_{\mathrm{u}}}{V_{1}} \phi_{1} \phi_{2} \chi_{12} \\
& -\frac{V_{\mathrm{u}}}{V_{1}} \phi_{1} \phi_{2} \phi_{3}\left(\frac{\delta \chi_{13}}{\delta \phi_{2}}\right)_{\phi_{3}}-\frac{V_{\mathrm{u}}}{V_{2}} \phi_{2}^{2} \phi_{3}\left(\frac{\delta \chi_{23}}{\delta \phi_{2}}\right)_{\phi_{3}} \\
& +\left[\frac{V_{3}}{V_{1}} \phi_{1}\left(\frac{\delta \chi_{13}}{\delta \phi_{3}}\right)_{\phi_{2}}+\frac{V_{3}}{V_{2}} \phi_{2}\left(\frac{\delta \chi_{23}}{\delta \phi_{3}}\right)_{\phi_{2}}\right] \\
& -\phi_{3}\left(\phi_{1}+\phi_{2}\right)=0
\end{aligned}
$$

where $V_{i}$ is the molar volume of component $i ; V_{\mathrm{u}}$ the volume of polymer per mole repeating unit; $\chi_{12}, \chi_{13}$ 
and $\chi_{23}$ are interaction parameters for the ternary system. $\chi_{13}$ and $\chi_{23}$ are expressed as functions of $\phi_{2}$ and $\phi_{3}$, whereas $\chi_{12}$ is assumed to be function only of $h_{2}=\phi_{2} /\left(1-\phi_{3}\right)$ (i.e., $\chi_{12}$ are equal along lines of constant $\phi_{1} / \phi_{2}$ ) [7,9]. In Eq. (5), parameters $A, B$ and $C$ are related to the heat of fusion of polymer $\left(\Delta H_{\mathrm{f}}\right)$ and the heat capacities of the polymer in the solid $\left(C_{\mathrm{c}}\right)$ and melt $\left(C_{\mathrm{m}}\right)$ states. If $C_{\mathrm{c}}$ and $C_{\mathrm{m}}$ can be assumed to be linear functions of temperature, namely $C_{\mathrm{m}}=a+b T$, and $C_{\mathrm{c}}=c+d T$, then one obtains $A=a-c, B=b-d$, and $C=\Delta H_{\mathrm{f}}-A T_{\mathrm{m}}-B T_{\mathrm{m}} / 2$. Given the values of the interaction parameters, Eq. (5) can be solved to obtain the crystallization curve. On the other hand, one can determine the interaction parameters by using a regression method, provided that experimental phase equilibrium data are available. The details of computation are shown by Cheng [26].

\subsection{Binary interaction parameters $\chi_{12}$ and $\chi_{13}$}

Binary interaction parameter between component 1 (water) and 2 (DMSO), $\chi_{12}$, may be determined using vapour liquid equilibrium data $[9,24,25] . \chi_{12}$ is expressed in terms of composition of the liquid phase,

$$
\begin{aligned}
\chi_{12}= & {\left[-x_{1} \ln \left(x_{1}+\Lambda_{12} x_{2}\right)-x_{2} \ln \left(\Lambda_{21} x_{1}+x_{2}\right)\right.} \\
& \left.+x_{1} \ln x_{1}+x_{2} \ln x_{2}-x_{1} \ln \phi_{1}-x_{2} \ln \phi_{2}\right] / x_{1} \phi_{2}
\end{aligned}
$$

where $x_{i}$ is the mole fraction of component $i$ in the liquid phase and $\Lambda_{i j}$ the Wilson's parameter. Assuming that $\chi_{12}$ has the functional form suggested by Koningsveld and Kleintjens [27], parameters $a, b$, and $c$ in Eq. (7) can be found by least square regression.

$\chi_{12}=a-\frac{b}{1-c \phi_{2}}$

For a ternary system, following Yilmaz and McHugh [25], $\chi_{12}$ is assumed to be function only of $\phi_{2} /\left(1-\phi_{3}\right)$. Thus, ternary $\chi_{12}$ is obtained by replacing $\phi_{2}$ with $h_{2}=\phi_{2} /\left(1-\phi_{3}\right)$ in Eq. (7).

Binary interaction parameter between component 1 (water) and 3 (polymer), $\chi_{13}$, may be determined using data from equilibrium sorption experiments [28]. The following equation is employed:

$$
\ln \frac{p}{p^{o}}=\ln \phi_{1}+\left(1-\frac{1}{x_{n}}\right) \phi_{3}+\chi_{13} \phi_{3}^{2}
$$

where $p^{\circ}$ is the saturation pressure of the nonsolvent and $x_{n}$ the degree of polymerization of the polymer.

\section{Experimental}

\subsection{Material}

EVAL copolymer containing ca. 56 mole percent vinyl alcohol (intrinsic viscosity $=0.87 \mathrm{dL} / \mathrm{g}$, $M_{\eta}=56000 \mathrm{~g} / \mathrm{mol}$ ) was kindly supplied by Kuraray Co., Ltd., Japan. Intrinsic viscosity was measured with a Ubbehode viscometer at $30 \pm 0.1{ }^{\circ} \mathrm{C}$ in a $85 \mathrm{wt} \%$ phenol aqueous solution [29]. Dimethylsulfoxide (DMSO, Baker Analyzed, reagent grade, $d=$ $1.096 \mathrm{~g} / \mathrm{ml}$ ) was used as received. Water was double distilled and deionized before use.

\subsection{Gelation and liquid-liquid demixing boundaries}

The gelation and liquid-liquid demixing boundaries were determined by cloud point method described in a previous publication [9]. A specific amount of EVAL copolymer (dried in oven at $50^{\circ} \mathrm{C}$ ) was mixed with a suitable amount of DMSO and sealed in a Teflonlinked bottle $(20 \mathrm{ml})$. The mixture was then blended for two days at room temperature until polymer was completely swelled. To this solution was added a known quantity of nonsolvent. Local precipitation may be observed. This mixture ( $10 \mathrm{~g}$ total) was agitated in a roll mill at room temperature for several hours and then put in an oven until a clear homogeneous solution was obtained. To examine the effect of the equilibrium phase behavior, the solution was placed in an isothermal thermostat which was maintained at $25^{\circ} \mathrm{C}, 45^{\circ} \mathrm{C}, 65^{\circ} \mathrm{C}$ and $85^{\circ} \mathrm{C}$, respectively, for a period of three days. With different nonsolvent content, three types of phase-separated results may be observed: (i) the solution precipitated into a translucent or a white gel (e.g., Fig. 1(b)); (ii) solution becomes a clear liquid phase coexistent with a white solid (e.g., Fig. 1(c)); (iii) solution undergoes liquidliquid phase transition into two clear liquid phases. The equilibrium gelation point (case (i)) was identified as the composition at which a sharp increase in turbidity was observed by a turbidity bridge (Digital turbidimeter, Orbeco-Hellige, USA). Case (ii) indicates a systems having concurrence of gelation and 


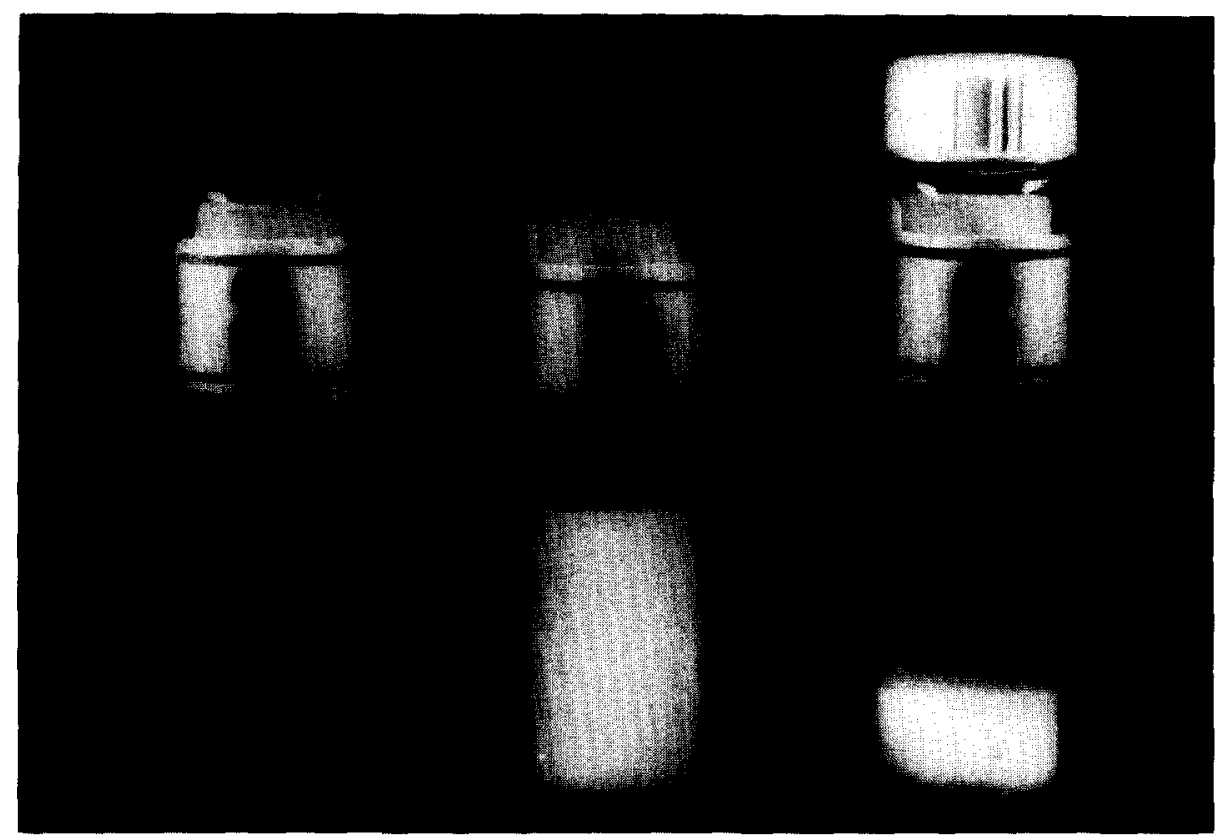

Fig. 1. Observed phase separation of EVAL in water-DMSO solutions. (a) A one-phase homogeneous solution; (b) solution precipitated into a gel; (c) solution becomes a clear liquid coexistent with a gel.

liquid-liquid demixing. For case (iii), which was observed only for low polymer concentrations and high temperatures, the location of binodal in the ternary phase diagram was determined.

\subsection{Characterization of gels}

The thermal property of the equilibrium crystalline EVAL gels were studied using a differential scanning calorimeter (DuPont 2200) for the temperature range of $25^{\circ} \mathrm{C}$ to the melting points of the gels. Dried gels of EVAL were prepared by removing solvent and nonsolvent in a cryogenic evaporator (Lubconco lyophilizer, USA). The morphology of the dried gel was examined using a scanning electron microscope at $20 \mathrm{kV}$.

\subsection{Membrane preparation and characterization}

Membranes with porous structures were prepared using direct immersion-precipitation method. A homogeneous solution composed of EVAL and DMSO was spread uniformly on a glass plate (ca. $220 \mu \mathrm{m})$. This solution was directly immersed into a bath wherein precipitation takes place and the membrane solution becomes a white solid. The formed membrane was soaked in water to remove DMSO and then in acetone and dried in open air. SEM photomicrographs of the dried membranes were taken in different views.

\subsection{Equilibrium absorption of water of amorphous EVAL}

EVAL pellets were fused in a mechanical press at 6000 psi and $185^{\circ} \mathrm{C}$ The EVAL melt was subsequently quenched in liquid nitrogen to form amorphous EVAL films. Equilibrium water absorption was measured by Cahn Counter balance at $25^{\circ} \mathrm{C}$.

\section{Results and discussion}

\subsection{Phase behavior of water-DMSO-EVAL at $25^{\circ} \mathrm{C}$}

In Fig. 2, phase diagram of water-DMSO-EVAL at $25^{\circ} \mathrm{C}$ is shown. The data points (denoted by $\boldsymbol{A}$ ) represent the experimental gelation boundary. A solu- 


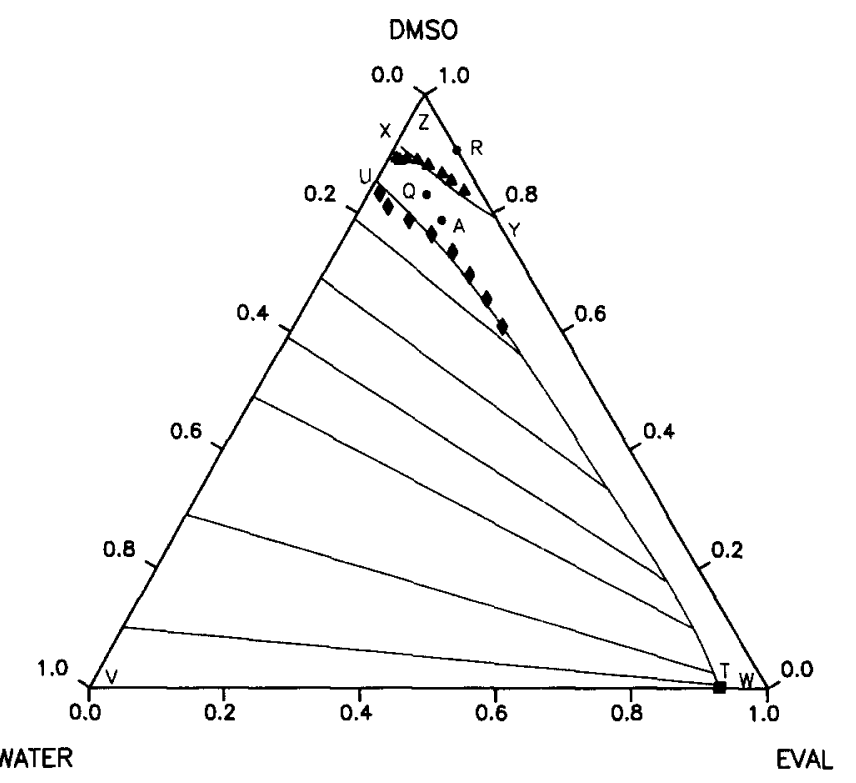

Fig. 2. Phase diagram of water-DMSO-EVAL at $25^{\circ} \mathrm{C}$. $\mathbf{A}$ : measured gel points. $\bullet$ : measured binodal points. $\square$ : measured equilibrium absorption of water by EVAL. Line XY: computed crystallization line. Region VUT: computed liquid-liquid demixing envelop.

tion above this boundary is homogenous and transparent as shown in Fig. 1(a). EVAL polymer may be dissolved in DMSO to form a good solution in which the EVAL relaxes and behaves like an expanded coil. As water (nonsolvent) is added to this solution, the strong interaction between water and DMSO reduces the solvation character of EVAL in DMSO. When the solution is brought within the gelation miscibility gap, the water-DMSO mixture acts as a precipitant and the EVAL coil collapse and gelation occurs. The solution becomes an opaque paste-like gel consisting of small aggregates dispersed in a liquid phase, as shown in Fig. 1(b). A DSC thermogram of this gel (point A and Fig. 2) is shown in Fig. 1(b). A DSC thermogram of this gel (point A in Fig. 2) is shown in Fig. 3 for the process of heating the gel from $25^{\circ} \mathrm{C}$ to $90^{\circ} \mathrm{C}$ at a rate of $10^{\circ} \mathrm{C} / \mathrm{min}$. A broad melting peak with a minimum at $54^{\circ} \mathrm{C}$ was observed, which corresponds to the melting of crystallites of EVAL copolymer in the gel. Films of EVAL copolymer were prepared by freeze drying of the gels and the morphology of these crystalline solids were examined using SEM. A typical result is shown in Fig. 4. As can be observed that the EVAL film is consisted of interconnected globular particles. These particles were formed initiated by crystallization nucleation of EVAL in DMSO-water mixture. The

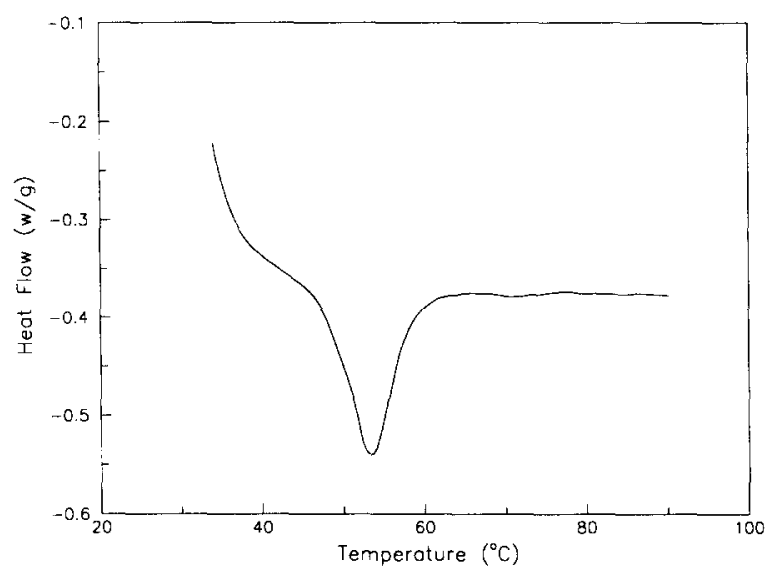

Fig. 3. DSC thermogram of a crystalline gel (point A in Fig. 2). $T_{\mathrm{m}}=54^{\circ} \mathrm{C}$; Enthalpy of fusion, $\Delta H=9.021 \mathrm{~J} / \mathrm{g}$.

nuclei grow radiality into the shape of spheres. Induction of polymer crystallization from a solution by a nonsolvent has also been reported by Cheng et al. $[5,6,9]$ for the systems water-formic acid-Nylons. For such ternary systems, they pointed out the possibility of a sequential development of liquid-liquid phase separation and crystallization in an direct immersionprecipitation process for the formation of porous membranes. After contact of the polymer solution 


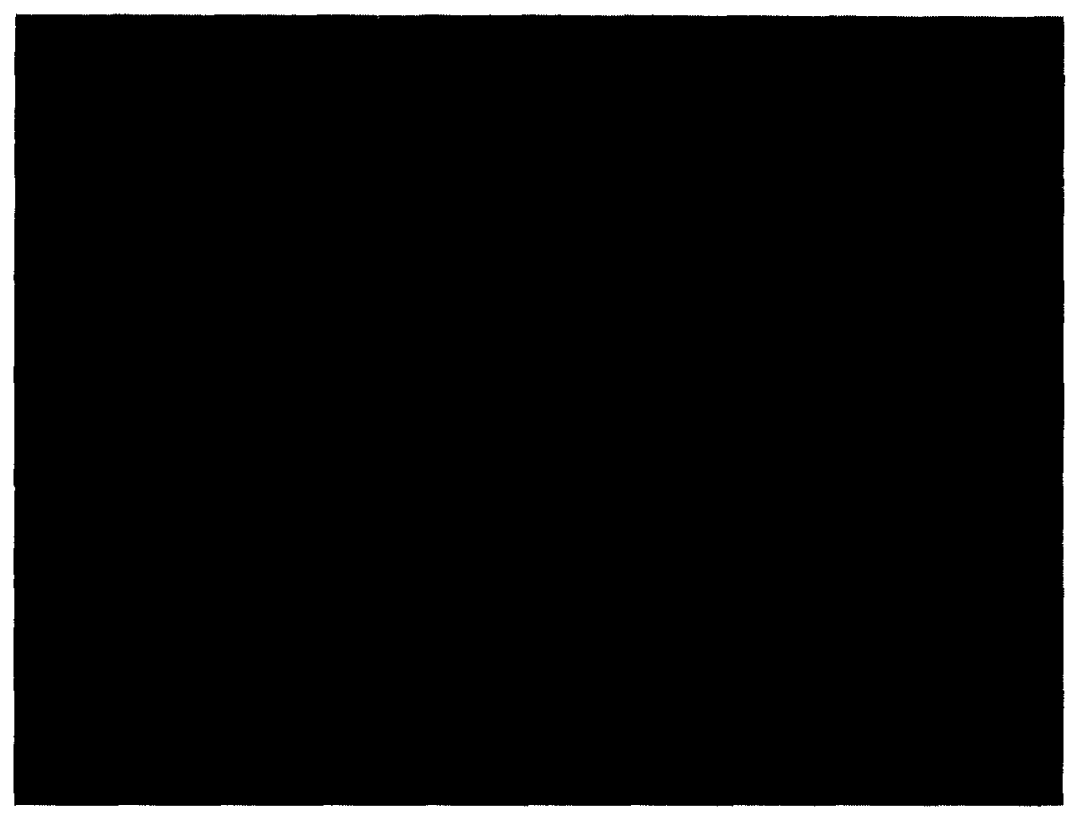

Fig. 4. SEM photomicrograph of EVAL solid prepared from a gel (point A in Fig. 2).

with nonsolvent, liquid-liquid phase separation takes place first in most cases and then followed by crystallization. Consequently, an asymmetric structure characterized by a skin and cellular pores was often observed. In order to prepare a uniform microporous membrane with a crystallization dominant morphology (characterized by interlocked crystalline particles), liquid-liquid phase separation has to be sufficiently suppressed. In this regard they suggested to use a soft bath that contained substantial amount of solvent and a dope that was in a state of incipient crystallization. Bulte et al. [8] investigated the mechanism of formation of Nylon-46 membranes had also reported observation of membrane structures formed by liquid-liquid demixing and crystallization.

The equilibrium gelation data at $25^{\circ} \mathrm{C}$ were used with Eq. (5) to obtain the best fitted crystallization isotherm, line $\mathrm{XY}$, in Fig. 2. It appears that line $\mathrm{XY}$ matches very closely the experimental data points. The best fitted interaction parameters $\chi_{23}$, was determined and shown in Table 1. The concentrationdependent interaction parameter for water-DMSO binary pair, $\chi_{12}$, was determined separately using vapour-liquid equilibrium data from literature [30]. Nonlinear regression was then used with Eq. (7) to find the parameters $a, b$, and $c$, and the results are given in Table 1. At $25^{\circ} \mathrm{C}$, EVAL copolymer was reported to absorb $6.7 \mathrm{wt} \%$ water in equilibrium condition [29]. Using Eq. (8), interaction parameter, $\chi_{13}$, was obtained. Other physical constants for computation are given in Table 2. The heat of fusion at the melting temperature, $\Delta H$, and the heat capacities for EVAL copolymer in the solid and melt states were determined using DSC measurements.

The best-fitted interaction parameters between DMSO and EVA, $\chi_{23}$, are negative for all compositions. This suggest an exothermic mixing between DMSO and EVAL over the entire concentration range. Therefore, it is not appropriate to use solubility parameters, which use positive enthalpic terms, to describe the interactions between these components. Highly negative interaction parameters between solvent and polymer were also reported by Cheng [9] and Bulte [31] for the formic acid-Nylon systems. It was claimed that hydrogen bonding and protonation effects $[32,33]$ are responsible for the strong interaction forces between these molecules. The interaction parameter between water and EVAL was treated as being concentration independent since Eq. (8) and equilibrium absorption data were used. Constant $\chi_{13}$ was also employed by Yilmaz [25], Altena [24] and Cheng [9] in different systems to compute the phase 
Table 1

Physical constants for EVAL copolymer

\begin{tabular}{llllll}
\hline$T_{\mathrm{m}}$ & $\begin{array}{l}C_{\mathrm{m}} \\
(\mathrm{J} / \mathrm{mol} \mathrm{K})\end{array}$ & $\begin{array}{l}C_{\mathrm{c}} \\
(\mathrm{J} / \mathrm{mol} \mathrm{K})\end{array}$ & $\begin{array}{l}\Delta H \\
(\mathrm{~kJ} / \mathrm{kg})\end{array}$ & $\begin{array}{l}\rho \\
\left(\mathrm{g} / \mathrm{cm}^{3}\right)\end{array}$ & $\begin{array}{l}M \\
(\mathrm{~g} / \mathrm{mol})\end{array}$ \\
\hline 163.1 & $0.0405 T+87.54$ & $0.4749 T-83.59$ & 68.62 & 1.17 & 56,000 \\
\hline
\end{tabular}

$T_{\mathrm{m}}$ : melting temperature; $C_{\mathrm{c}}$ : crystalline heat capacity; $C_{\mathrm{m}}$ : amorphous heat capacity; $\Delta H$ : enthalpy of fusion; $\rho$ : density.

Table 2

Summary of interaction parameter data

\begin{tabular}{ll}
\hline Binary system & Interaction parameter, $\chi_{i j}$ \\
\hline Water (1)/DMSO (2) & $-1.302+1.08 /\left(1.0-0.86 \phi_{2}\right)$ \\
Water (1)/EVAL (3) & 1.956 \\
DMSO (2)/EVAL (3) & $-1.2+0.2 \phi_{3}$ \\
\hline
\end{tabular}

$\varphi_{\mathrm{i}}$ : volume fraction of component $i(i=1$ : water, $i=2$ : DMSO, $i=3$ : EVAL).

separation boundaries. Very good match between calculated and measured results were reported by these authors. In the current case, the value of $\chi_{13}$ was found to be 1.956 . This large positive value is consistent with the general understanding that water is a harsh nonsolvent for EVAL copolymer.

In this report, the liquid-liquid phase equilibrium boundary was determined by solving Eqs. (1-3) and the results were compared with those obtained from cloud point measurements of case (ii) situation. The interaction parameters and physical constants employed in binodal computations are given in Tables 1 and 2. The calculated binodal (line VUT) and tie lines are shown in Fig. 2 together with the experimental liquid-liquid demixing data points (denoted by $\bullet$ ). These points represent the composition at which liquid-liquid demixing first occurs in a series of samples with increasing amount of nonsolvent (i.e., the amount of liquid phase above the white gel is diminutive). Line VTU appears to agree with the measured data points over a wide concentration range. This implies that it is possible to measure the binodal with reasonable accuracy even in systems with crystallization. The data point at $\phi_{2}=0$ in Fig. 2 (denoted by $\square$ ) depicts the measured equilibrium water absorption of amorphous EVAL at $25^{\circ} \mathrm{C}$. The calculated result (i.e., point $T$ ) fits perfectly the experimental data. This further assure a reliable binodal calculation.

\subsection{Membrane formation with crystallization and liquid-liquid demixing}

The binodal and crystallization boundaries divide the phase diagram at $25^{\circ} \mathrm{C}$ (Fig. 2) into three parts. Any solution above the crystalline line (region $\mathrm{XYZ}$ ) is single-phase and homogeneous. The region defined by the binodal and the crystallization lines (approximately region TUXYW in Fig. 2 and called the "gel region", hereinafter) is metastable exclusively which respect to crystallization. Any composition in this region will precipitate into a gel induced by crystallization. The region within the binodal phase envelop (region VUT) is metastable with respect to both liquid-liquid phase separation and crystallization. Therefore, both types of phase transformation are anticipated in this region. In an immersion-precipitation process for preparing EVAL copolymer membranes, a uniform dope solution (usually in region $\mathrm{XYZ}$ ) is allowed to contract with a nonsolvent. As a result of the exchange of solvent and nonsolvent in the membrane solution, the composition may be brought across the binodal into region VUT wherein both types of precipitation are likely to occur. In this case, the morphology of the formed membranes depends upon the relative rate of crystallization and liquid-liquid demixing. Because initiation of liquid-liquid demixing is in general more rapid than nucleation of polymer crystallization which requires a de-solvation and rearrangement of polymer [5,6], liquid-liquid demixing is kinetically favored and often commences the precipitation process. The liquid microdroplets form surrounded by polymer-rich phase and grow into the cellular pores in the final membrane. Since the polymer-rich phase is always supersaturated with respect to crystallization, EVAL copolymer eventually crystallizes to form the solid matrix of the final membrane. However, crystallization occurs at a late stage at which liquid pores have fully developed, the morphology is shown in Fig. 5. This membrane is prepared by 


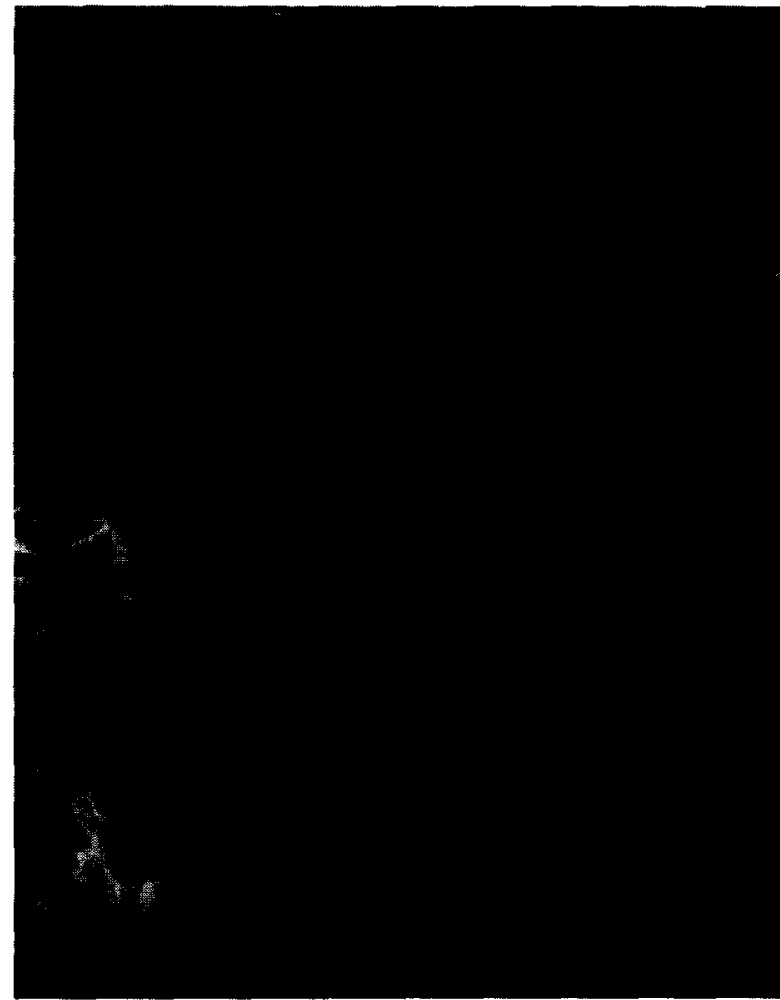

Fig. 5. SEM photomicrograph of an EVAL membrane. Dope composition (point R in Fig. 2): $\phi_{1}=0.1, \phi_{2}=0.905, \phi_{3}=0.094$. Bath: 70 wt\% DMSO in water.

immersing a good dope solution (point $\mathrm{R}$ in Fig. 2) into an aqueous bath containing $70 \mathrm{wt} \%$ DMSO. Liquid-liquid demixing (i.e., the circular cellular pores) is evident in this membrane. Vestige of crystallization can only be seen at the cell walls in the form of particles. In contrast to Fig. 5, a membrane with a structure dominated by crystallization may be prepared using the same bath and an incipient dope with respect to crystallization (point $Q$ in Fig. 2). The structure of this membrane is shown in Fig. 6. It resembles the structure of the dried gel shown in Fig. 4. The crystalline particles are largely spherical. Cellular pores resulted from liquid-liquid phase separation is not evident in this membrane.

The tie lines of this system (Fig. 2) are very steep and all have negative slopes. This is frequently the case in which membrane solution precipitates into asymmetric structures. As the membrane solution contacts the nonsolvent bath, a concentrated layer forms just beneath the membrane-bath interface. The composition of this layer is at the end of the tie line. Because this layer is rich in polymer, it is mechanically strong and thus cannot be broken by liquid-liquid demixing and/or crystallization occurred underneath it. As a result, a thin and continuous dense skin is often observed in the final membrane.

\subsection{Effect of temperature on the miscibility envelops}

In Fig. 7, experimental data points for the onset of gelation of EVAL solutions are given for a series of temperatures, $25^{\circ} \mathrm{C}, 45^{\circ} \mathrm{C}, 65^{\circ} \mathrm{C}$ and $85^{\circ} \mathrm{C}$, respectively. It can be seen that liquid-liquid demixing and gelation dominate different regions of the phase diagram for different temperatures. The isotherms at $25^{\circ} \mathrm{C}$ and $45^{\circ} \mathrm{C}$ represent the compositions of the phases that are in equilibrium with the EVAL copolymer crystalline gels. At $45^{\circ} \mathrm{C}$, some of the gels which were formed at $25^{\circ} \mathrm{C}$ gaining enough thermal energy fuse and become homogeneous solutions. The one-phase region, thus, becomes larger than that at $25^{\circ} \mathrm{C}$. It is interesting to notice that the gelation line at $45^{\circ} \mathrm{C}$ shifts toward nonsolvent-polymer axis in a manner roughly parallel to the gelation line at $25^{\circ} \mathrm{C}$. Pure liquid-liquid demixing was not observed at $45^{\circ} \mathrm{C}$. As the temperature is increased to $65^{\circ} \mathrm{C}$, both liquid-liquid demixing (in the absence of crystallization) and crystallization-induced gelation were observed and these boundaries intersect at $\phi_{2}=0.74$. Liquid-liquid demixing occurs at low polymer concentrations $\left(\phi_{3}<\mathrm{ca} .0 .12\right)$, in which the original precipitated gel separates into two homogeneous solutions in equilibrium, whose compositions are located at the binodal (tie line ends). For high polymer concentrations $\left(0.3>\phi_{3}>0.12\right)$, crystallization dominates and equilibrium gels are formed. As in the case of gelation at $45^{\circ} \mathrm{C}$, this gelation line moves downward roughly parallel with the gelation line at $25^{\circ} \mathrm{C}$. The calculated binodal at $25^{\circ} \mathrm{C}$ is also shown in Fig. 7 . Unlike the gelation boundary which changes substantially with temperature, the binodal is affected only slightly for the range between $25^{\circ} \mathrm{C}$ and $85^{\circ} \mathrm{C}$. A phenomenon which is comparable to the current case has been reported by Stokes and Berghmans [34,35] for the binary poly(vinyl alcohol)/ethylene glycol system (nonsolvent was not used). In a cooling 


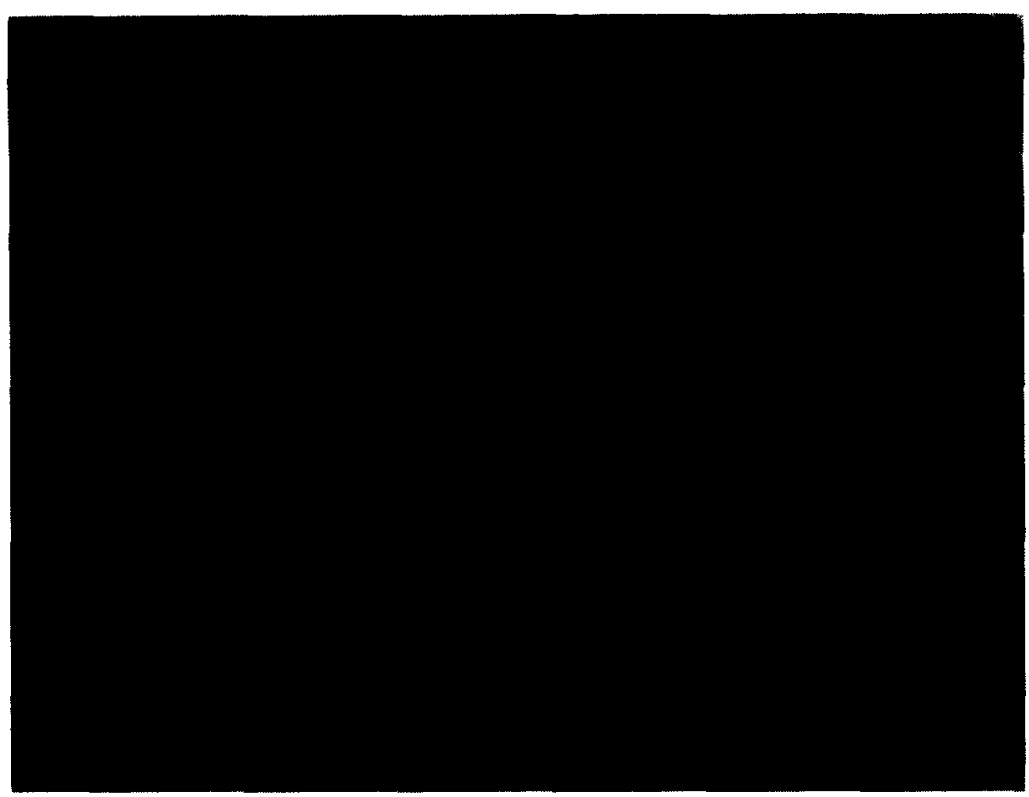

Fig. 6. SEM photomicrograph of an EVAL membrane. Dope composition (point Q in Fig. 2): $\phi_{1}=0.082, \phi_{2}=0.831, \phi_{3}=0.087$. Bath: 70 wt $\%$ DMSO in water.

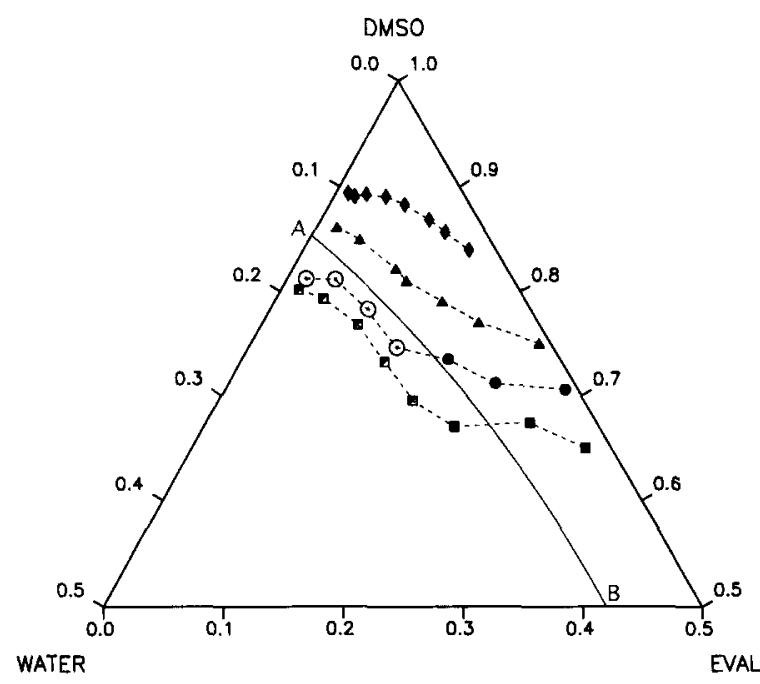

Fig. 7. Phase diagram of water-DMSO-EVAL at various temperatures. $\$$ : measured gelation data at $25^{\circ} \mathrm{C}$. $\mathbf{\Delta}$ : measured gelation data at $45^{\circ} \mathrm{C}$. $\odot$ : measured binodal at $65^{\circ} \mathrm{C}$. $:$ : measured gelation data at $65^{\circ} \mathrm{C}$. $\mathrm{D}$ : measured binodal at $85^{\circ} \mathrm{C}$. $\square$ : measured gelation data at $85^{\circ} \mathrm{C}$. Line $\mathrm{AB}$ : computed binodal at $25^{\circ} \mathrm{C}$.

process, this system was found to exhibit both crystallization and liquid-liquid demixing, and because liquid-liquid phase demixing is less sensitive to temperature changes, these two phases transition bound- aries intersect each other in certain concentrations. This suggests that phase transition of a binary polymer-solvent system caused by temperature variation can alternatively be brought to happen by adjusting the quantity of nonsolvent in the polymer solution. In a similar way, instances of transition from gelation to liquid-liquid demixing for ternary systems was also observed by Wijmans et al. for poly(2,6-dimethyl-1,4phenylene oxide) in various solvent-nonsolvent pairs [7].

\section{Conclusions}

Phase transformation boundaries of the ternary system, water-DMSO-EVAL copolymer, were obtained over a wide temperature range. The following points may be drawn from the determined phase diagram of this system.

(1) At temperature lower than $45^{\circ} \mathrm{C}$, the phase diagram contains three regions (i.e., a single-phase region, a gel region, and a binodal region) defined by the crystallization and liquid-liquid demixing isotherms. In the gel region, precipitation occurs only by nucleation and growth of polymer crystallites. In the gel region, liquid-liquid demixing initiates phase 
separation and crystallization of the polymer-rich phase follows.

(2) At higher temperatures, the binodal and crystallization lines intersect. Thus, a fourth region exists in which liquid-liquid demixing in the absence of crystallization dominates.

(3) The normally unobservable amorphous phase behavior of crystalline EVAL in aqueous DMSO solutions can be determined by interaction parameters that are fitted with crystallization data.

\section{Acknowledgements}

The authors thank the National Science Council of Taiwan, the Repubic of China, for their financial support of this research (NSC 85-2216-E-002-002 and 85-2216-E-032-001).

\section{References}

[1] H. Strathmann, in D.R. Lloyd (Ed.), Material Science of Synthetic Membrane, ACS Symposium Series, 1985.

[2] D.M. Koenhen, M.H.V. Mulder and C.A. Smolders, Phase separation phenomena during the foundation of asymmetric membranes, J. Appl. Polym. Sci., 21 (1977) 199-215.

[3] M.H.V. Mulder, J. Oude Hendrikman, J.G. Wijmans and C.A. Smolders, A rationale for the formation of asymmetric pervaporation membranes, J. Appl. Polym. Sci., 30 (1985) 2805-2820.

[4] A.J. Reuvers, F.W. Altena and C.A. Smolders, Demixing and gelation behavior of ternary cellulose acetate solutions, J. Polym. Sci., Polym. Phys., 24 (1986) 793-804.

[5] L.P. Cheng, A.W. Dwan and C.C. Gryte, Membrane formation by isothermal precipitation in polyamide-formic acid-water systems I. Description of membrane morphology, J. Polym. Sci., Polym. Phys., 33 (1995) 211-222.

[6] L.P. Cheng, A.W. Dwan and C.C. Gryte, Membrane formation by isothermal precipitation in polyamide-formic acid-water systems II. Precipitation dynamics, J. Polym. Sci,, Polym. Phys., 33 (1995) 223-235.

[7] J.G. Wijmans, H.J.J. Rutten and C.A. Smolders, Phase separation phenomena in solutions of poly(2,6-dimethyl-1,4phenyleneoxide) in mixtures of trichloroethylene, 1-octanol, and methanol: relationship to membrane formation, J. Polym. Sci., Polym. Phys., 23 (1985) 1941-1955.

[8] A.M.W. Bulte, B. Folkers, M.H.V. Mulder and C.A. Smolders, Membranes of semicrystalline aliphatic polyamide Nylon 4,6: Foundation by diffusion-induced phase separation, J. Appl. Polym. Sci., 50 (1993) 13-26.

[9] L.P. Cheng, A.W. Dwan and C.C. Gryte, Isothermal phase behavior of Nylon- $6,-66$, and -610 polyamides in formic acid-water systems, J. Polym. Sci., Polym. Phys., 32 (1994) 1183-1190.

[10] S. Yamashita, S. Nagata and K. Takakura, US Patent 4,134,837, 1979.

[11] Y. Sakurada, A. Sueoka and M. Kawahashi, Blood purification device using membranes derived from poly(vinyl alcohol) and copolymer of ethylene and vinyl alcohol, Polym. J., 19 (1987) 501-513.

[12] L.W. Chen and T.H. Young, EVAL membranes for blood dialysis, Makromol., Chem. Macromol. Symp., 33 (1990) 183-199.

[13] T.H. Young and L.W. Chen, A diffusion-controlled model for wet-casting membrane formation, J. Membrane Sci., 59 (1991) 169-181.

[14] T.H. Young, Effects of temperature on wet-casting membrane formation from EVAL copolymers, Biomed. Eng.: Appl. Basis Commun., 4 (1992) 71-78.

[15] T.H. Young and L.W. Chen, Roles of bimolecular interaction and relative diffusion rate in membrane structure control, $\mathrm{J}$. Membrane Sci., 83 (1993) 153-166.

[16] T.H. Young and L.W. Chen, Pore formation mechanism of membranes from phase inversion process, Desalination, 103 (1995) 233-247.

[17] N. Guerra, L. Barbani, L. Lazzeri, L. Lelli, M. Palla and C. Rizzo, The activation of human plasma prekallikrein as a hemocompatibility test for biomaterials. II. Contact activation by EVAL and EVAL-SMA copolymers, J. Biomaterials Sci., Polym. Ed., 4 (1993) 643-652.

[18] J.C. Chaloupka, F. Vinuela, H.V. Vinters and J. Robert, Technique feasibility and histopathologic studies of ethylene vinyl alcohol copolymer (EVAL) using a swine endovascular embolization model, Am. J. Neuroadiol,, 15 (1994) 11071115.

[19] J.J. Lewandowski, P.S. Malchesky, M. Zborowski and Y. Nose, Evaluation of a miniature blood glucose sensor, Trans. Am. Soc. Artif. Intern. Organs, 34 (1988) 255-258.

[20] K. Nakamae, M. Kameyama and T. Matsumoto, Elastic moduli of the crystalline regions in the direction perpendicular to the chain axis of ethylene-vinyl alcohol copolymers, Polym. Eng. Sci., 19 (1979) 572-578.

[21] A. Apicella, H.B. Hopfenberg and S. Piccarolo, Low temperature thermal aging of ethylene vinyl alcohol copolymers, Polym. Eng. Sci., 22 (1982) 382-387.

[22] M. Modell and R. Reid, Thermodynamics and Its Applications, 2nd edn., Prentice-Hall, Englewood Cliffs, NJ, 1983.

[23] P.J. Flory, Principles of Polymer Chemistry, Cornell University Press, Ithaca, York, NY, 1953.

[24] F.W. Altena and C.A. Smolders, Calculation of liquid-liquid phase separation in a ternary system of a polymer in a mixture of a solvent and a nonsolvent, Macromolecules, 15 (1982) 1491-1497.

[25] L. Yilmaz and A.J. McHugh, Analysis of nonsolventsolvent-polymer phase diagrams and their relevance to membrane formation modeling, J. Appl. Polym. Sci., 31 (1986) 997-1018.

[26] L.P. Cheng, Doctoral Dissertation, Columbia University, New York, 1993. 
[27] R. Koningsveld and L.A. Kleintjens, Macromolecules, 4 (1971) 637.

[28] D. Bonner, Vapour-liquid equilibria in concentrated polymer solutions, J. Macromol. Sci.-Revs. Macromol. Chem., Cl3(2) (1975) 263-319.

[29] T. Okaya and K. Ikari, in C.A. Finch (Ed.), Polyvinyl Alcohol Developments, Wiley, Chichester, 1992.

[30] S.Y. Lam and R.L. Benoit, Some thermodynamic properties of the dimethylsulfoxide-water and propylene carbonatewater system at $25^{\circ} \mathrm{C}$, Can. J. Chem., 52 (1974) 718-722.

[31] A.M.W. Bulte, Doctoral Dissertation, Twente University, Netherlands, 1994.
[32] M.E. Epstein and A.J. Rosenthal, Spinning of polyamides from sulfuric acid solution, Textile Res. J., 36(9) (1966).

[33] G.W. Yao, R.P. Burford, A.G. Fane, C.J.D. Feil and R.M. McDonogh, Hydrolysis and other phenomena affecting structure and performance of polyamide 6 membrane, J. Appl. Polym. Sci., 34 (1987) 2399-2408.

[34] W. Stokes, H. Berghmans, P. Moldenaers and J. Mewis, Thermoreversible gelation of solutions of poly(vinyl alcohol), Polym. J., 20 (1988) 361-369.

[35] W. Stokes and H. Berghmans, Phase behavior and gelation of solutions of poly(vinyl alcohol), J. Polym. Sci., Polym. Phys., 29 (1991) 609-617. 